\title{
Detection of SARS-CoV-2 variant 501Y.V2 in Comoros Islands in
}

\section{January 2021 [version 1; peer review: 2 approved]}

\section{Charles Agoti (Di) 1*, George Githinji (D)1*, Khadija S. Mohammed (D1,} Arnold W. Lambisia (D1) , Zaydah R. de Laurent (D1, Maureen W. Mburu1, Edidah M. Ong'era1, John M. Morobe (D1, Edward Otieno (D1), Hamza Abdou Azali², Kamal Said Abdallah³, Abdoulaye Diarra33 , Ali Ahmed Yahaya4, Peter Borus ${ }^{5}$, Nicksy Gumede Moeletsi ${ }^{4}$, Dratibi Fred Athanasius ${ }^{4}$, Benjamin Tsofa(D1, Philip Bejon (1D1,6, D. James Nokes (D1,7, Lynette Isabella Ochola-Oyier1

\footnotetext{
${ }^{1}$ Kenya Medical Research Institute (KEMRI) - Wellcome Trust Research Programme, Kilifi, Kenya, KILIFI, KILIFI, 230-80108, Kenya

2Institut National de Recherche pour l'Agriculture, la Pêche et l'Environnement (INRAPE), Comoros, Comoros

${ }^{3}$ Malaria National Referral Laboratory, Comoros; World Health Organisation (WHO) Country Office., Comoros, Comoros

${ }^{4}$ WHO/Africa Regional Office (AFRO), Brazzaville, Congo, Democratic Republic

${ }^{5}$ WHO Country Office, Nairobi, Kenya

${ }^{6}$ Nuffield Department of Medicine, Oxford University, United Kingdom (UK), Oxford, UK

7University of Warwick, Coventry, UK

* Equal contributors
}

V1 First published: $28 \mathrm{Jul}$ 2021, 6:192

https://doi.org/10.12688/wellcomeopenres.16889.1

Latest published: $28 \mathrm{Jul}$ 2021, 6:192

https://doi.org/10.12688/wellcomeopenres.16889.1

\section{Abstract}

Background. Genomic data is key in understanding the spread and evolution of SARS-CoV-2 pandemic and informing the design and evaluation of interventions. However, SARS-CoV-2 genomic data remains scarce across Africa, with no reports yet from the Indian Ocean islands.

Methods. We genome sequenced six SARS-CoV-2 positive samples from the first major infection wave in the Union of Comoros in January 2021 and undertook detailed phylogenetic analysis.

Results. All the recovered six genomes classified within the 501Y.V2 variant of concern (also known as lineage B.1.351) and appeared to be from 2 sub-clusters with the most recent common ancestor dated $30^{\text {th }}$ Oct-2020 (95\% Credibility Interval: 06 th Sep-2020 to $10^{\text {th }}$ Dec-2020). Comparison of the Comoros genomes with those of 501Y.V2 variant of concern from other countries deposited into the GISAID database revealed their close association with viruses identified in France and Mayotte (part of the Comoros archipelago and a France, Overseas Department).

\section{Open Peer Review \\ Approval Status \\ 1 \\ 2 \\ version 1 \\ 28 Jul 2021

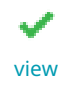 \\ view \\ 1. I-Ching Sam ID, University of Malaya, Kuala Lumpur, Malaysia \\ 2. Aminu S. Jahun (D), University of Cambridge, Addenbrooke's Hospital Level 5, Cambridge, UK}

Any reports and responses or comments on the article can be found at the end of the article. 
Conclusions. The recovered genomes, albeit few, confirmed local transmission following probably multiple introductions of the SARSCoV-2 501Y.V2 variant of concern during the Comoros's first major COVID-19 wave. These findings demonstrate the importance of genomic surveillance and have implications for ongoing control strategies on the islands.

Keywords

SARS-CoV-2, 501Y.V2, Comoros

Corresponding author: Charles Agoti (CNyaigoti@kemri-wellcome.org)

Author roles: Agoti C: Conceptualization, Formal Analysis, Methodology, Project Administration, Supervision, Visualization, Writing Original Draft Preparation, Writing - Review \& Editing; Githinji G: Conceptualization, Formal Analysis, Funding Acquisition, Methodology, Project Administration, Supervision, Writing - Original Draft Preparation, Writing - Review \& Editing; S. Mohammed K: Investigation, Writing - Review \& Editing; W. Lambisia A: Investigation, Writing - Review \& Editing; R. de Laurent Z: Investigation, Writing - Review \& Editing; W. Mburu M: Investigation, Writing - Review \& Editing; M. Ong'era E: Investigation, Writing - Review \& Editing; M. Morobe J: Investigation, Writing - Review \& Editing; Otieno E: Data Curation, Writing - Review \& Editing; Abdou Azali H: Writing - Review \& Editing; Said Abdallah K: Writing - Review \& Editing; Diarra A: Writing - Review \& Editing; Ahmed Yahaya A: Writing - Review \& Editing; Borus P :Writing - Review \& Editing; Gumede Moeletsi N: Writing - Review \& Editing; Fred Athanasius D: Writing - Review \& Editing; Tsofa B: Project Administration, Writing - Review \& Editing; Bejon P: Project Administration, Writing - Review \& Editing; James Nokes D: Funding Acquisition, Project Administration, Writing - Review \& Editing; Isabella Ochola-Oyier L: Project Administration, Supervision, Writing Review \& Editing

Competing interests: No competing interests were disclosed.

Grant information: This work was supported by grants to Prof. James Nokes from Wellcome Trust [220985] and National Institute for Health Research (NIHR) fundings (project reference 17/63/82) and a second NHIR grant to Prof. Mark Woolhouse (project reference 16/136/33). NHIR grants use UK aid from the UK Government and the UK Foreign Commonwealth and Development Office to support global health research. The views expressed in this publication are those of the authors and not necessarily those of NIHR, the Department of Health and Social Care, Foreign Commonwealth and Development Office, Wellcome Trust, or the UK government. The funders had no role in study design, data collection and analysis, decision to publish, or preparation of the manuscript.

Copyright: @ 2021 Agoti C et al. This is an open access article distributed under the terms of the Creative Commons Attribution License, which permits unrestricted use, distribution, and reproduction in any medium, provided the original work is properly cited.

How to cite this article: Agoti C, Githinji G, S. Mohammed K et al. Detection of SARS-CoV-2 variant 501Y.V2 in Comoros Islands in January 2021 [version 1; peer review: 2 approved] Wellcome Open Research 2021, 6:192

https://doi.org/10.12688/wellcomeopenres.16889.1

First published: 28 Jul 2021, 6:192 https://doi.org/10.12688/wellcomeopenres.16889.1 


\section{Introduction}

Although Comoros, an island country in the Indian ocean, detected its first case of SARS-CoV-2 on $30^{\text {th }}$ April 2020, it experienced its first major SARS-CoV-2 outbreak in January 2021 i.e., 10 months later $^{1}$. By $28^{\text {th }}$ February 2021, Comoros had 3,571 laboratory-confirmed SARS-CoV-2 infections, 2,748 (76.9\%) of which were confirmed after $1^{\text {st } J a n u a r y ~} 2021$.

Genomic surveillance has been key in understanding the introduction, spread, and evolution of SARS-CoV-2 pandemic into countries since its emergence in late 2019 in China and in informing the design and evaluation of interventions ${ }^{2-4}$. Towards the end of 2020, in widely different geographical locations globally, three SARS-CoV-2 variants of concern (Alpha, Beta, and Gamma) emerged that appeared to be considerably more transmissible and with potential to facilitate immune escape or cause more severe disease than the prior SARS$\mathrm{CoV}-2$ variants $^{5-7}$. The three variants possessed several defining amino acid changes, most of them occurring within the immunogenic spike $(\mathrm{S})$ protein $^{8}$. The $\mathrm{S}$ protein contains the domain that binds the virus to the human host cell receptor and is a key target for several vaccines ${ }^{9}$. Here, we investigated if the variants of concern had a role in the rising number of SARS-CoV-2 cases in the Union of Comoros in January 2021.

\section{Methods}

An earlier version of this article can be found on bioRxiv (DOI: https://doi.org/10.1101/2021.04.08.21254321).

\section{Ethical statement}

The SARS-CoV-2 genomes were generated as part of a regional collaborative COVID-19 public health rapid response. The whole genome sequencing study protocol was reviewed and approved by the Scientific and Ethics Review Committee (SERU), Kenya Medical Research Institute (KEMRI), Kenya (SERU \#4035). Individual patient consent was not required by the committee for the use of these samples for sequencing as a part of the public health emergency response.

\section{Study site and samples}

The samples analysed had been collected between $5^{\text {th }}$ and $11^{\text {th }}$ January 2021 in the Union of Comoros, specifically from two islands, Ngazidja and Mohéli (Table 1). A total of 11 positive nasopharyngeal/oropharyngeal swab samples were sent to KEMRI-Wellcome Trust Programme (KWTRP) in Kilifi, Kenya, for genome analysis. KWTRP is one of the 12 designated WHO-AFRO /Africa-CDC specialized and regional reference laboratories for SARS-CoV-2 sequencing in Africa ${ }^{10}$. Sample size was determined by the sequence available from the public health response.

\section{Laboratory procedures}

On receiving the samples on the $16^{\text {th }}$ and $17^{\text {th }}$ January 2021, viral RNA was extracted using the QIAamp Viral RNA Mini kit (52906, Qiagen, Hilden, Germany) following the manufacturer's instructions and analysed using the Sansure Biotech Novel Coronavirus (2019-nCoV) Nucleic acid Diagnostic real-time RT-PCR commercial kit (S3102E, Sansure Inc., China) which targets the nucleocapsid (N) and ORF1ab regions. Nine of the 11 samples were confirmed as SARS-CoV-2 positive by both gene targets (cycle threshold $(\mathrm{Ct})<38.0$ ). We proceeded to sequence six samples that had a $\mathrm{Ct}$ value of $\leq 29.0$. Samples with $\mathrm{Ct}$ above 29.0 were excluded because we observed in our laboratory that they frequently fail the downstream quality control steps before sequencing due to possession of low viral titres. The RNA was first reverse transcribed using the LunaScript ${ }^{\circledR}$ RT SuperMix Kit (E3010, New England Biolabs Inc., Germany) then amplified using the Q5® Hot Start High-Fidelity 2X Master Mix (NEB M0494; New England Biolabs Inc., Germany) along with the ARTIC nCoV-2019 version 3 primers $^{11}$. The resultant amplicons were taken forward for library preparation and MinION (Mk1B) (Oxford Nanopore Technology, Oxford) sequencing. The six samples were processed alongside 17 other samples from coastal Kenya to make a batch of 23 samples.

\section{Data analysis}

The MinION (https://nanoporetech.com/products/minion) sequencing read-outs (fast5 files) were base-called using the Guppy basecaller 4.4.0 fast model and subsequently demultiplexed. Consensus genomes assembled using a SARS-CoV-2 ARTIC Network Bioinformatics pipeline ${ }^{11}$. A threshold of 20x coverage was required for a base to be included in the consensus genome otherwise it was masked to $\mathrm{N}$. We retrieved 75 random Beta variant sequences from the GISAID database (selected by downloading all the Beta variant sequences that were available on $28^{\text {th }}$ February 2021 and then using an inhouse python script to sub-sample). These data were then aligned with the Union of Comoros genomes (now available on GISAID) using MAFFT v.7.313 (https://mafft.cbrc.jp/alignment/software/). The alignment was manually inspected in SEAVIEW v 4.6 .4 (http://doua. prabi.fr/software/seaview) to spot any obvious misalignments. We reconstructed maximum likelihood (ML) phylogeny using IQTREE v.1.6.12 (http://www.iqtree.org/). Branch support was evaluated using 1,000 bootstrap iterations. The presence of a molecular clock signal was inspected in TempEst v1.5.3 (http:// tree.bio.ed.ac.uk/software/tempest/). Linear regression of rootto-tip genetic distances against sampling dates were plotted in $\mathrm{R}$ v4.0.2 (https://www.r-project.org/). We inferred time-scaled phylogenies in BEAST V1.10.4 (https://beast.community/) under the $\mathrm{HKY}+\mathrm{G}$ substitution model with an uncorrelated relaxed molecular clock assumption. BEAST Markov chain Monte Carlo (MCMC) runs were set to run 50 million steps with sampling after every 2,500 steps $^{12}$. The BEAST run output was analysed in Tracer v1.7.1 (http://tree.bio.ed.ac.uk/software/tracer/). Dated maximum clade credibility phylogeny was inferred using TreeAnnotator v1.10.4 (https://beast.community/treeannotator after discarding $10 \%$ as burn-in and visualized using FigTree v1.4.4 (http://tree.bio.ed.ac.uk/software/figtree/).

\section{Results}

We assembled $>80 \%$ of the SARS-CoV-2 genome from each of the six sequenced samples (Table 1). The recovered genomes were classified into the lineage B.1.351 using the Pangolin 


\begin{tabular}{|c|c|c|c|c|c|c|}
\hline $\begin{array}{l}\text { Study } \\
\text { ID }\end{array}$ & $\begin{array}{l}\text { GISAID } \\
\text { Accession } \\
\text { Number }\end{array}$ & Sex & Island & $\begin{array}{l}\text { Date } \\
\text { sampling }\end{array}$ & Ct N gene, ORF1ab & Genome length ${ }^{\pi}, \%$ \\
\hline $\mathrm{C} 001$ & EPI_ISL_1323658 & Male & Ngazidja & Jan-2021 & $24.06,26.42$ & $26960,90 \%$ \\
\hline $\mathrm{COO2}$ & EPI_ISL_1323659 & Female & Ngazidja & Jan-2021 & $23.8,26.46$ & $26638,89 \%$ \\
\hline $\mathrm{COOH}^{\#}$ & - & Female & Ngazidja & - & 35.64, undetermined & - \\
\hline $\mathrm{COOH}^{\#}$ & - & Female & Ngazidja & - & $\begin{array}{l}\text { Undetermined, } \\
\text { undetermined }\end{array}$ & - \\
\hline C005 & EPI_ISL_1323660 & Female & Ngazidja & Jan-2021 & $21.4,23.8$ & $26640,89 \%$ \\
\hline $\mathrm{C} 006^{¥, \#}$ & - & - & & Jan-2021 & $29.98,34.28$ & - \\
\hline $\mathrm{COO7}$ & EPI_ISL_1323661 & Male & Mohéli & Jan-2021 & $24.21,27.33$ & $26358,88 \%$ \\
\hline $\mathrm{CO08}$ & - & Male & Mohéli & Jan-2021 & $33.39,35.79$ & - \\
\hline C009 & EPI_ISL_1323662 & Female & Mohéli & Jan-2021 & $16.41,18.59$ & $26638,89 \%$ \\
\hline $\mathrm{C} 010$ & - & Female & Mohéli & Jan-2021 & $31.8,35.35$ & - \\
\hline $\mathrm{C} 011$ & EPI_ISL_1323663 & Female & Mohéli & Jan-2021 & $18.77,21.26$ & $26400,88 \%$ \\
\hline
\end{tabular}

*Intensive care unit case.

${ }^{\pi}$ Complete SARS-CoV-2 genome is 29,903 nucleotides long (Wuhan 2019 reference, accession number: NC_ 045512.2).

\#Experienced missing data/demographic details in the submitted forms and were not sequenced.

toolkit v2.3.0 $0^{13}$. The genomes possessed six of the eight Beta variant defining amino acid changes in the $\mathrm{S}$ protein (i.e., L18F, D80A, D215G, K417N, D614G, and A701V) plus a known three amino acid deletion at positions 243-245. Two additional defining amino acid changes (E484K and N501K) which were unconfirmed fell within a region that was not sequenced due to PCR amplicon drop-off. Our findings and confirmation of the presence of the SARS-CoV-2 Beta variant in Comoros samples was conveyed to Union of Comoros authorities on the $22^{\text {nd }}$ January via the WHO-AFRO office to inform public health actions.

The six Union of Comoros sequences differed only at three nucleotide positions: A13192G (1 genome), T23560C (2 genomes), and G27505T (1 genome). Compared to the Wuhan 2019 reference (Accession number: NC_45512.2), the Union of Comoros genomes had 21-22 nucleotide substitutions that translated into 16-17 amino acid changes. A time-scaled MCC phylogenetic tree of these sequences revealed that the Union of Comoros genomes formed a monophyletic group together with genomes from Mayotte (which is part of the Comoros archipelago and a French Overseas department) and France (Figure 1). This group diverged into two sub-clusters with the most recent common ancestor dated $30^{\text {th }}$ Oct-2020 (95\%CI: 06 ${ }^{\text {th }}$ Sep-2020 to $10^{\text {th }}$ Dec-2020).

\section{Discussion}

We provide evidence of circulation of the Beta variant during the first major SARS-CoV-2 epidemic peak in the Union of Comoros. The Beta variant was first identified in South Africa and had been reported in 41 countries as of $19^{\text {th }}$ February 2021. Initial data suggested that this variant exhibits up to 6-fold reduction in neutralization activity by post-vaccination sera or convalescent sera from individuals infected by prior variants $^{14}$. Thus, finding this variant in Union of Comoros is concerning since it has potential to overcome pre-existing immunity derived from natural infection or vaccination.

Understanding the extent of spread of this variant in Union Comoros is limited by the low number of cases sequenced. As of $28^{\text {th }}$ February 2021, the number of new cases in the Union of Comoros had considerably declined after peaking in midJanuary ${ }^{1}$. Comparison of the Union of Comoros genomes with genomes from across the globe found a close relation with those from the neighbouring Mayotte, a French Overseas Department. Mayotte detected its first case of SARS-CoV-2 on 10th March 2020 and experienced its first major SARS-CoV-2 outbreak from mid-January 2021 to mid-March 2021 thus overlapping with the Union of Comoros outbreak. 721 SARS-CoV-2 genomes were available on GISAID database from Mayotte as of $23^{\text {rd }}$ April 2021 and the majority (52\%) were classified 
a.

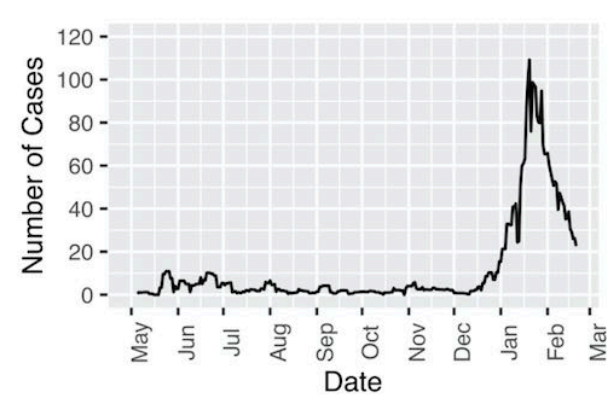

C.
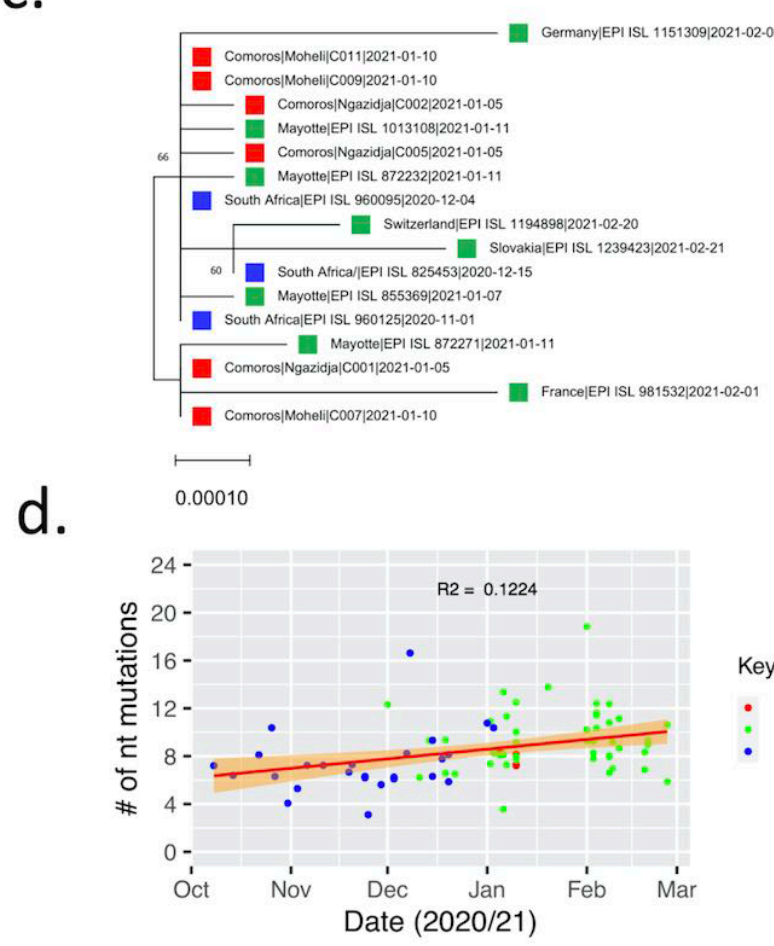

Key b.

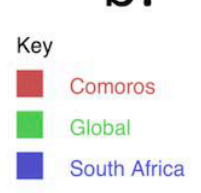

Key

South Africa
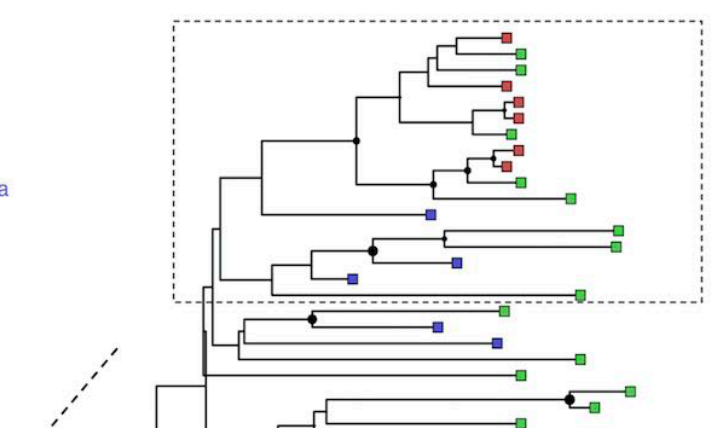

- Comoros Global South Africa

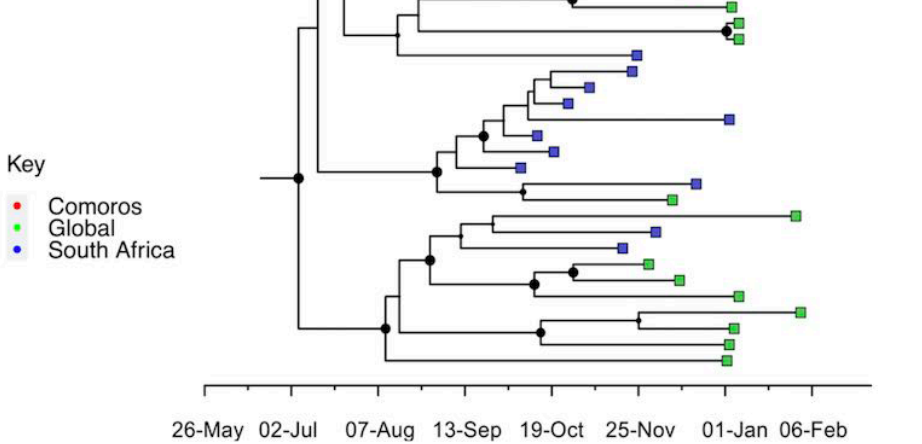

Figure 1. Genomic diversity of SARS-CoV-2 in Comoros Islands. a. Smoothed daily number of cases reported in Comoros Islands since the observation of the first case on 30 $0^{\text {th }}$ April 2020 up to $19^{\text {th }}$ February 2020. $\mathbf{b}$. time-scaled phylogenetic tree showing the placement of the six samples from Comoros (coloured in red) relative to other Beta variant sequences from around the globe coloured in green. Those from South Africa are coloured blue. $\mathbf{d}$. a zoomed in Maximum Likelihood tree of the clade containing the newly sequenced Comoros genomes. c. Root-to-tip divergence among the analysed 81 Beta genomes.

as Beta variant ${ }^{15}$. Our study demonstrates how continental genomic surveillance using the Sequencing Laboratory Network for Covid-19 can be utilised to inform response to the SARS-CoV-2 pandemic.

\section{Data availability}

\section{Underlying data}

The six genomes have been deposited in GISAID (https://www. gisaid.org/), Accession numbers EPI_ISL_1323658 - EPI_ISL_ 1323663 and can be accessed following registration and login. The Comoros daily case data for the period between April 2020 and February 2021 was obtained and is freely available from our in Our World in Data database (https://ourworldindata.org/coronavirus/country/comoros).

Harvard Dataverse. Replication Data for: Detection of SARSCoV-2 variant 501Y.V2 in Comoros Islands in January 2021. https://doi.org/10.7910/DVN/NDOIQ2.

This project contains the following underlying data:

- CAgoti_Comoros_Genome_Codebook.pdf. (Data table-1. tab structure description). 
- CAgoti_Comoros_Genome_Readme.txt. (Readme file for data table-1.tab).

- DATA_Table-1.tab. (This dataset is part of the continuous genomic surveillance of SARS-CoV-2 courtesy of the Laboratory Sequencing Network created by WHO-AFRO and AFRICA-CDC. This dataset contains results of samples positive for SARS-CoV-2 by real-time PCR for the period 5th January 2020-10th January 2020).

- DATA_Table.tab. (This dataset is part of the continuous genomic surveillance of SARS-CoV-2 courtesy of the Laboratory Sequencing Network created by WHOAFRO and AFRICA-CDC. This dataset contains results of samples positive for SARS-CoV-2 by real-time PCR for the period $5^{\text {th }}$ January $2020-10^{\text {th }}$ January 2020 ).
- gisaid_hcov-19_acknowledgement_table_2021_06_ 12_18.pdf. (This dataset acknowledges authors from the originating laboratories responsible for obtaining the specimens, as well as the submitting laboratories where the genome data were generated and shared via GISAID, on which this research is based).

Data are available under the terms of the Creative Commons Attribution 4.0 International license (CC-BY 4.0).

\section{Acknowledgements}

We thank the WHO-AFRO and the WHO Kenya and Comoros country offices for facilitating sharing of the positive samples that were sequenced. Further, we thank all laboratories that have shared SARS-CoV-2 sequence data on GISAID.
1. Worldmeter: World / Countries / Comoros / Coronavirus. Coronavirus, 2021; [cited 2021 23-Mar-2021].

Reference Source

2. Bugembe DL, Kayiwa J, Phan MVT, et al.: Main Routes of Entry and Genomic Diversity of SARS-CoV-2, Uganda. Emerg Infect Dis. 2020; 26(10): 2411-2415. PubMed Abstract | Publisher Full Text | Free Full Text

3. Oude Munnink BB, Nieuwenhuijse DF, Stein M, et al.: Rapid SARS-CoV-2 wholegenome sequencing and analysis for informed public health decisionmaking in the Netherlands. Nat Med. 2020; 26(9): 1405-1410. PubMed Abstract | Publisher Full Text

4. Worobey M, Pekar J, Larsen BB, et al.: The emergence of SARS-CoV-2 in Europe and North America. Science. 2020; 370(6516): 564-570. PubMed Abstract | Publisher Full Text | Free Full Text

5. Davies NG, Jarvis CI, CMMID COVID-19 Working Group, et al.: Increased mortality in community-tested cases of SARS-CoV-2 lineage B.1.1.7. Nature. 2021; 593(7858): 270-274.

PubMed Abstract | Publisher Full Text

6. Tegally $\mathrm{H}$, Wilkinson $\mathrm{E}$, Giovanetti $\mathrm{M}$, et al.: Emergence of a SARS-CoV-2 variant of concern with mutations in spike glycoprotein. Nature. 2021 Reference Source

7. Faria NR, Mellan TA, Whittaker C, et al.: Genomics and epidemiology of a novel SARS-CoV-2 lineage in Manaus, Brazil. medRxiv. 2021; 2021.02.26.21252554.

PubMed Abstract | Publisher Full Text | Free Full Text

8. Zimmer K: A Guide to Emerging SARS-CoV-2 Variants. In: The scientist. 2021. Reference Source
9. Ortuso F, Mercatelli D, Guzzi PH, et al.: Structural genetics of circulating variants affecting the SARS-CoV-2 spike/human ACE2 complex.J Biomol Struct Dyn. 2021; 1-11.

PubMed Abstract | Publisher Full Text | Free Full Text

10. WHO: COVID-19 genome sequencing laboratory network launches in Africa. 2020 Reference Source

11. Tyson JR, James P, Stoddart D, et al.: Improvements to the ARTIC multiplex PCR method for SARS-CoV-2 genome sequencing using nanopore. bioRxiv. 2020; 2020.09.04.283077.

PubMed Abstract | Publisher Full Text | Free Full Text

12. Agoti CN, Munywoki PK, Phan MVT, et al.: Transmission patterns and evolution of respiratory syncytial virus in a community outbreak identified by genomic analysis. Virus Evol. 2021; 3(1): vex006. PubMed Abstract | Publisher Full Text | Free Full Text

13. Rambaut A, Holmes EC, O'Toole Á, et al.: A dynamic nomenclature proposal for SARS-CoV-2 lineages to assist genomic epidemiology. Nat Microbiol. 2020; 5(11): 1403-1407.

PubMed Abstract | Publisher Full Text | Free Full Text

14. Wu K, Werner AP, Moliva JI, et al.: mRNA-1273 vaccine induces neutralizing antibodies against spike mutants from global SARS-CoV-2 variants. bioRxiv. 2021; 2021.01.25.427948.

PubMed Abstract | Publisher Full Text | Free Full Text

15. Agoti CN, Githinji G, Mohammed KS, et al:: Replication Data for: Detection of SARS-CoV-2 variant 501YV2 in Comoros Islands in January 2021. Harvard Dataverse, V2, UNF: 6:fbZB4rfcSnOpndHaOqL4UA== [fileUNF]. 2021. http://wwww.doi.org/10.7910/DVN/NDOIQ2 


\section{Open Peer Review}

\section{Current Peer Review Status:}

\section{Version 1}

Reviewer Report 11 January 2022

https://doi.org/10.21956/wellcomeopenres.18631.r47738

(C) 2022 Jahun A. This is an open access peer review report distributed under the terms of the Creative Commons Attribution License, which permits unrestricted use, distribution, and reproduction in any medium, provided the original work is properly cited.

\section{Aminu S. Jahun}

Division of Virology, Department of Pathology, University of Cambridge, Addenbrooke's Hospital Level 5, Cambridge, CB2 0QQ, UK

Agoti et al. report on the sequencing and analyses of six SARS-CoV-2 samples from the Comoros Islands, all of which were found to be of the B.1.351 lineage. The manuscript is clear and concise, and their data will be of significant interest to officials in-charge of public health responses, and to the global scientific community.

I only have a few minor comments as follows:

I noticed that there was no mention of the inclusion of negative controls in the sequencing assays, and how the presence of mapped reads in the negative controls, if any, was addressed. This is particularly relevant given the high similarity between the six samples.

Including the assembly pipeline version, and indicating which tool was used in generating the consensus sequence (nonopolish or medaka) may be useful for others who may be interested in including these data in future analyses

In Table 1, perhaps just 'genome coverage', +/- number of Ns, may be more appropriate than 'genome length'.

Figure 1 legend labels 'c' and 'd' are swapped (typo?).

Also potential typo - 1st sentence, 4th paragraph on page 4 of the pdf, 'Union of Comoros' instead of 'Union Comoros'.

Is the work clearly and accurately presented and does it cite the current literature? Yes

Is the study design appropriate and is the work technically sound? 
Are sufficient details of methods and analysis provided to allow replication by others? Partly

If applicable, is the statistical analysis and its interpretation appropriate?

Not applicable

Are all the source data underlying the results available to ensure full reproducibility?

Yes

Are the conclusions drawn adequately supported by the results?

Yes

Competing Interests: No competing interests were disclosed.

Reviewer Expertise: SARS-CoV-2 sequencing and analyses

I confirm that I have read this submission and believe that I have an appropriate level of expertise to confirm that it is of an acceptable scientific standard.

Reviewer Report 01 December 2021

https://doi.org/10.21956/wellcomeopenres.18631.r47089

(C) 2021 Sam I. This is an open access peer review report distributed under the terms of the Creative Commons Attribution License, which permits unrestricted use, distribution, and reproduction in any medium, provided the original work is properly cited.

\section{I-Ching Sam}

Department of Medical Microbiology, Faculty of Medicine, University of Malaya, Kuala Lumpur, Malaysia

This is a brief report based on 6 SARS-CoV-2 sequences from the Union of Comoros, which were the only Comoros sequences available in GISAID at the time of publication (July 2021). The samples were from January 2021, during the first major COVID-19 wave. Standard methods were used. Sequences were shown to be of the beta variant of concern and clustered together, similar to sequences from nearby Mayotte and South Africa. Although limited by the small number of sequences and lack of epidemiological data, the report is of local and regional interest as there is little/no molecular epidemiology data from Comoros.

There are no major issues.

\section{Minor issues:}

More relevant details of the setting may help increase understanding of the significance of the findings. For example, case incidence per 100,000 during this wave, incidence in Ngazidja and Mohéli islands, travel restrictions and likely sources of importation, etc.

Is there any further epidemiological data for these 6 cases? e.g. travel history, contact with 
each other.

Fig 1a legend has a typo, should be "19th Feb 2021".

Fig 1 legend, (d) and (c) are the wrong way round.

Is the work clearly and accurately presented and does it cite the current literature?

Yes

Is the study design appropriate and is the work technically sound?

Yes

Are sufficient details of methods and analysis provided to allow replication by others?

Yes

If applicable, is the statistical analysis and its interpretation appropriate?

Yes

Are all the source data underlying the results available to ensure full reproducibility?

Yes

Are the conclusions drawn adequately supported by the results?

Yes

Competing Interests: No competing interests were disclosed.

Reviewer Expertise: Medical virology, respiratory viruses

I confirm that I have read this submission and believe that I have an appropriate level of expertise to confirm that it is of an acceptable scientific standard. 